\title{
Erfassung des familienmedizinischen Interventionsbedarfs durch eine direkte Befragung von Patienten mit einer psychischen Störung
}

\section{Assessment of Family Based Intervention Needs by a Direct Survey of Patients with a Psychiatric Disorder}

Autoren

Katharina Zogas, Georg Juckel, Paraskevi Mavrogiorgou

Institut

Klinik für Psychiatrie, Psychotherapie und Präventivmedizin, LWL-Universitätsklinikum der Ruhr-Universität Bochum

\section{Schlüsselwörter}

psychiatrische Störungen, Kinder psychisch kranker Eltern, Interventionsbedarf, Früherkennung

\section{Keywords}

psychiatric disorders, children of mentally ill parents, need of intervention, psychoeducation

Bibliografie

DOI https://doi.org/10.1055/a-1084-2037

Online-Publikation: 17.1.2020 | Psychiat Prax 2020; 47:

128-134

(c) Georg Thieme Verlag KG Stuttgart · New York

ISSN 0303-4259

\section{Korrespondenzadresse}

Prof. Dr. med. Georg Juckel, Klinik für Psychiatrie, Psychotherapie und Präventivmedizin, LWL-Universitätsklinikum der Ruhr-Universität Bochum, Alexandrinenstraße 1,

44791 Bochum

georg.juckel@rub.de

\section{ZUSAMMENFASSUNG}

Hintergrund Die Belastung von Familien psychisch Kranker, besonders von deren Kindern, wird in der Literatur wenig beleuchtet. Gerade die Versorgungssituation Minderjähriger während eines stationären Aufenthalts eines Elternteils und die damit verbundenen Sorgen der Eltern sind wenig erforscht.

Anliegen Erfassung der Versorgungssituation von Kindern und des familiären Interventionsbedarfs stationär behandelter Eltern mit psychischer Erkrankung.

Methodik Im Rahmen einer prospektiven Studie wurden 100 Patienten mit den Hauptdiagnosen affektive Störung, psychische und Verhaltensstörung durch psychotrope Substanzen und Persönlichkeitsstörung mittels eines standardisierten Interviews und Fragebogens näher untersucht.

Ergebnisse In der Mehrheit der Fälle haben die behandelnden Psychiater kein Wissen über die Versorgungssituation der Kinder ihrer Patienten. Der Großteil der Patienten gibt Sorgen um die Versorgung der Kinder während ihres stationären Aufenthalts an und wünscht sich eine Verbesserung der Hilfsmaßnahmen seitens der Klinik.

Schlussfolgerung Die Arzt-Patienten-Interaktion und Kommunikation sollte optimiert werden, damit Interventionsbedarfe gezielt erfasst und individuelle Hilfsmaßnahmen angeleitet werden können.

\section{ABSTRACT}

Background The burden on families of the mentally ill, especially on their children, is little explored in the literature. Especially the care situation of minors during a stationary stay of a parent and the related concerns of the parents are little researched.

Aim Assessment of the care situation of children and the need for family intervention of inpatient parents with mental illness.

Methods In a prospective study 100 patients with the main diagnoses affective disorder, mental and behavioral disorder by psychotropic substances and personality disorder were examined using a standardized interviews and questionnaire.

Results In the majority of cases psychiatrists don't know about the care situation of their patients' children. Most of the patients declare anxiety for the child's care while being in inpatient treatment. They desire an improvement of supporting measures.

Conclusions The interaction and communication between patient and psychiatrist should be improved to capture the need of intervention and to instruct individual supporting measures. 


\section{Einleitung}

Für psychische Erkrankungen wird aktuell von einer 12-MonatsPrävalenz von 27,7\% im Erwachsenenalter zwischen dem 18. und 79. Lebensjahr ausgegangen [1]. Das bedeutet, dass fast 18 Millionen in Deutschland lebende Menschen eine psychische Störung aufweisen, wobei nach Gühne et al. [2] bis zu 1 Million Deutsche unter einer schweren behandlungsbedürftigen psychischen Störung leiden. Die häufigsten psychischen Störungen sind dabei Angststörungen, affektive Erkrankungen sowie stoffgebundene Suchterkrankungen [1]. Trotz der hohen Zahl begibt sich mit 36\% nur ein relativ kleiner Teil der psychisch erkrankten Menschen in fachpsychiatrische bzw. psychotherapeutische Behandlung, wobei die Gründe hierfür vielgestaltig sind [3].

Erst in den letzten 20 Jahren wurde die Erforschung der Wechselwirkung im Zusammenhang zwischen intrafamiliärer Belastung und Krankheitsverlauf vor allem im Bereich der psychoedukativen Therapiekonzepte intensiviert. Die meisten der bisher dazu veröffentlichten Studien umfassen die Untersuchung von Patientenangehörigen, die an einer Erkrankung aus dem schizophrenen Formenkreis bzw. depressiven Störung [4] oder einer demenziellen Erkrankung (Übersichten: [5]) leiden. Nach Östmann et al. [6] sind die Gründe, die zu einer subjektiven Belastung der Angehörigen eines psychisch Erkrankten führen, nosologie-unspezifisch und auch nicht unbedingt von der Schwere der Symptomatik abhängig. Selbst ein Suizidversuch oder gegen Angehörige gerichtete Gewalt scheinen keinen Einfluss auf die subjektive Belastung zu haben. Die latente Sorge, selbst psychisch zu erkranken bzw. für diese Erkrankung genetisch prädisponiert zu sein, scheint hingegen einen größeren belastenden Einfluss auf den subjektiven Leidensdruck der Angehörigen zu haben [7].

Noch weniger wissenschaftlich erforscht sind die Auswirkungen der elterlichen psychischen Erkrankung auf die Entwicklung und Versorgung der Kinder trotz mittlerweile zahlreicher Hinweise, dass das Risiko für ein Kind, selber psychisch zu erkranken, deutlich höher ist, wenn ein Elternteil oder beide Elternteile psychisch erkrankt sind [8]. Interessant hierbei ist z. B. der wiederholt beschriebene Befund, dass die Behandler/ Therapeuten häufig kein bzw. unzureichendes Wissen über die familiären Hintergründe ihrer Patienten wie beispielsweise Anzahl von minderjährigen Kindern im gemeinsamen Haushalt und Versorgung dieser Kinder während der stationären Behandlungen des Patienten verfügen, obwohl standardisierte Fragen zur Familien- und Sozialanamnese zu den routinemäßigen Aufnahmemodalitäten gehören [9]. Im Gegensatz zu der Situation in Deutschland sind Ärzte z. B. in Finnland und Norwegen mittlerweile per Gesetz verpflichtet, sich aktiv nach Kindern und deren Lebenslage ihrer psychisch kranken Patienten zu erkundigen und bei Bedarf auf die bestehenden familienmedizinischen Projekte zu verweisen [10]. Ein Aspekt, warum gerade von Patienten, aber auch von Psychiatern Hilfseinrichtungen wie Jugendämter gemieden werden, ist die Furcht, ein Aufdecken des Hilfebedarfs könnte eine Kindeswegnahme provozieren [9].
Eltern wiederum, die für ihr psychisch krankes Kind (nicht selten lebenslang) sorgen, stehen vor der Herausforderung, das elterliche Pflichtgefühl und den Wunsch nach einem eigenen, selbstbestimmten Leben miteinander zu vereinen. Diesen Konflikt bewältigen sie meist alleine, da sie aufgrund von Scham und Schuldgefühlen mit Personen außerhalb der Familie wenig über eine psychische Erkrankung sprechen [11]. Betroffene Eltern empfinden nicht selten eine Macht- und Hilflosigkeit durch mangelnde Unterstützung der Gesundheitsbehörden, Schuldgefühle, Wut und Isolation, begleitet von einem tiefen Gefühl des Verlustes und Trauer [12]. Die besonderen Bedürfnisse der Eltern psychisch Erkrankter wurden ebenfalls lange Zeit nicht berücksichtigt. Dabei zeigt sich für den Kranken eindeutig ein positiver Effekt, wenn die Familie als Ressource in die Behandlung miteinbezogen wird. Eindrucksvoll konnten Pilling et al. [13] zeigen, dass bei Patienten mit einer Erkrankung aus dem schizophrenen Formenkreis bei Miteinbeziehung der familiären Bezugspersonen einerseits die Zahl der psychotischen Episoden sowie der stationären Aufnahmen sank und andererseits die Medikamenten-Compliance stieg.

Ziel unserer Untersuchung war es zunächst, durch die direkte Befragung von Patienten mit einer psychischen Erkrankung den familienmedizinischen Interventionsbedarf zu ermitteln. Gezielt sollte geklärt werden, ob psychisch kranke Menschen während des stationären Aufenthalts einen familienbezogenen Interventionsbedarf für sich erkennen und deklarieren, dabei Sorgen und Versorgungsprobleme deutlich gegenüber den Therapeuten formulieren und sich diesbezüglich und im Hinblick auf Hilfsangebote von diesen unterstützt fühlen. Hierbei sollte auch untersucht werden, inwieweit nosologie-spezifische Unterschiede bestehen und welche Rolle ein möglicher deklarierter Bedarf im Rahmen des Therapieprozesses einnimmt.

\section{Material}

\section{Patienten}

Das Untersuchungskollektiv umfasste insgesamt 100 Patienten, welche sich im Zeitraum vom 20.10.2017 bis 05.11.2018 aufgrund einer psychischen Störung nach ICD-10 in stationärer Behandlung in der Klinik für Psychiatrie, Psychotherapie und Präventivmedizin des LWL-Universitätsklinikums der Ruhr-Universität Bochum befanden.

Die Untersuchungspopulation setzt sich zusammen aus 50 Patienten mit der Hauptdiagnose einer affektiven Störung nach ICD-10 (F3.X), 25 Patienten mit einer nach ICD-10 bestehenden psychischen und Verhaltensstörung durch psychotrope Substanzen (F1.X) und 25 Patienten mit einer Persönlichkeitsstörung nach ICD-10 (F: 6.X). Insgesamt wurden 57 Frauen und 43 Männer mit mindestens einem zu versorgenden Kind befragt.

Ausschlusskriterien waren schwere somatisch-internistische und psychische Erkrankungen (z.B.organische Erkrankung des Gehirns) sowie das Nichtvorliegen der Einverständniserklärungen. Eine vorbestehende Medikation bei den Patienten stellte kein Ausschlusskriterium dar.

In einem durchschnittlich 60 Minuten andauernden semistrukturierten Interviewgespräch wurden neben den soziode- 
mografischen und klinischen Daten auch die Familiensituation sowie der Interventionsbedarf anhand des unten näher dargestellten Fragebogens erhoben.

Der Schweregrad der Erkrankung wurde anhand des CGI erfasst [14].

Das psychosoziale Funktionsniveau wurde mittels der Personal and Social Performance Scale untersucht (PSP; [15]).

Der hier erstmals verwendete Bochumer-Fragebogen zur Erfassung des familienmedizinischen Interventionsbedarfs durch eine direkte Befragung des Patienten (BOFREFI-DP) stellt eine modifizierte und zur direkten Befragung von Patienten adaptierte Version des umfangreichen Fragebogens zur Ermittlung des familienmedizinischen Interventionsbedarfs innerhalb aller LWL-Kliniken dar.

Für die vorliegende Untersuchung besteht die aktuelle Version des BOFREFI-DP aus insgesamt 45 Fragen, die mit Ausnahme der Fragen 27 (Zufriedenheit mit der aktuellen stationären Behandlung von 1: gar nicht - 5: sehr zufrieden) und 37 (Stärke der Sorgen bezüglich der Versorgung der Angehörigen 1: gar nicht - 5: sehr stark) jeweils mit ja oder nein und mit qualitativen Aussagen beantwortet werden konnten. Die ersten 26 Fragen dienten der Erhebung soziodemografischer, speziell familienbezogener und klinischer Daten. Es folgten Fragen zum Informationstransfer und Kenntnisstand des jeweiligen Behandlers über die familiäre Situation und evtl. vorliegenden Interventionsbedarf (9 Fragen), gefolgt von weiteren Fragen bezüglich der Darstellung von möglichen Hilfsangeboten durch den Therapeuten (3 Fragen). Gefragt wurden die Patienten auch, inwieweit sie sich mit ihren Sorgen bezüglich der Versorgung ihrer Kinder bzw. ihrer Familienangehörigen ernst genommen fühlten und inwieweit sie mit der Unterstützung diesbezüglich zufrieden waren (6 Fragen). Die letzte Frage stellte die Möglichkeit einer offenen Antwort bzw. persönliche Meinungsäußerung zum Thema Berücksichtigung des familiären Umfelds im Rahmen der stationären Behandlung dar.

Der Studieneinschluss der Patienten erfolgte entsprechend der Vorgaben der zuständigen Ethikkommission und der Deklaration von Helsinki (1975) nach ausführlicher Aufklärung und schriftlicher Einverständniserklärung. Für die Studie lag ein positives Ethikvotum der lokalen Ethikkommission (Nr.: 20155246) der Medizinischen Fakultät der Ruhr-Universität Bochum vor.

Zur Auswertung der Statistik wurde das Programm IBM SPSS Statistics 25 genutzt. Zur deskriptiven Analyse erfolgte die Berechnung der Mittelwerte und Standardabweichungen der erhobenen Daten. Die statistische Analyse erfolgte mittels parametrischer und/oder nonparametrischer Tests (t-Test, ANOVA und Pearson bzw. Spearman-Korrelationskoeffizienten). Werte von $\mathrm{p} \leq 0,05$ wurden als statistisch signifikant beziehungsweise Werte von $\mathrm{p} \leq 0,01$ als statistisch hochsignifikant gewertet.

\section{Ergebnisse}

\section{Soziodemografische und klinische Charakteristika}

Eine Darstellung der soziodemografischen und der allgemeinen klinischen Merkmale der gesamten Untersuchungspopulation findet sich in $\triangleright$ Tab. 1 .
- Tab. 1 Soziodemografische und klinische Charakteristika.

\begin{tabular}{|l|c|}
\hline $\begin{array}{l}\text { Alter, Mittelwert (SD) } \\
\text { Range }\end{array}$ & N=100 \\
\hline Geschlecht & $41,06(9,96)$ \\
Frauen, n & \\
F3.X & $57(57,0 \%)$ \\
F1.X & $28(28 \%)$ \\
F6.X & $8(8 \%)$ \\
Männer, n & $21(21 \%)$ \\
F3.X & $43(43,0 \%)$ \\
F1.X & $22(22 \%)$ \\
F6.X & $17(17 \%)$ \\
\hline
\end{tabular}

\section{Familienstand}

\begin{tabular}{|l|l}
\hline verheiratet/feste Partnerschaft, $n$ & $44(44 \%)$
\end{tabular}

keine Beziehung, $\mathrm{n} \quad 56(56 \%)$

Bildungsstand

\begin{tabular}{l|l} 
Abitur/Fachhochschulreife, $\mathrm{n}$ & $27(27 \%)$
\end{tabular}

mittlere Reife, $\mathrm{n} \quad 36(36 \%)$

Hauptschulabschluss, n $32(32 \%)$

Sonderschule, $\mathrm{n} \quad 2(2 \%)$

kein Schulabschluss, $n \quad 3(3 \%)$

\section{Berufsstatus}

abgeschlossene Berufsausbildung, $n \quad 63(63 \%)$

$\begin{array}{ll}\text { aktuell berufstätig, } n & \mathrm{n} 3(33 \%)\end{array}$

$\begin{array}{ll}\text { keine aktive Tätigkeit, } n & 67(67 \%)\end{array}$

\section{Diagnose}

affektive Störungen, $\mathrm{n} \quad 50(50 \%)$

$\begin{array}{ll}\text { F31 } 3(3 \%) & \end{array}$

F32 $3(3 \%)$

F33 $38(38 \%)$

sonstige $\quad 6(6 \%)$

Suchterkrankung (C2/Opiate), n $25(25 \%)$

F10 $24(24 \%)$

F11 $1(1 \%)$

\begin{tabular}{l|l} 
Persönlichkeitsstörung, n & $25(25 \%)$
\end{tabular}

F60.3 $24(24 \%)$

$\begin{array}{ll}\mathrm{F} 60.8 & 1(1 \%)\end{array}$

\section{Dauer der Erkrankung}

Mittelwert (SD) $\quad 15,76(10,56)$

Range 1-41 Jahre

CGI Mittelwert (SD) $\quad 4,9(0,63)$

PSP Mittelwert (SD)

$63,5(11,9)$

Bei $74 \%$ der Patienten handelte es sich aktuell um eine wiederholte stationäre Behandlung. Von den 100 Patienten waren zum Zeitpunkt der Untersuchung lediglich 14 Patienten medikamentenfrei, 86\% erhielten eine Psychopharmakotherapie: Am häufigsten erhielten die Patienten eine Kombinationsbehandlung, bestehend aus einem Antidepressivum (z. B. SSRI) und einem sedierenden Antipsychotikum. Bei 67\% der untersuchten Patienten lag eine positive Familienanamnese für psychische Erkrankungen vor, bei 24 Patienten bei mehr als einem Familienangehörigen. Bei 12 \% der befragten Patienten lag eine psychische Erkrankung beim leiblichen Kind vor. Als häufigste psychische Erkrankungen bei den Familienangehörigen wurden Depressionen ( $n=36)$ mit und ohne zusätzlicher Alkoholabhängigkeit $(n=16)$ angegeben. 
- Tab. 2 Familiäre Situation der Untersuchungspopulation ( $N=100)$.

\begin{tabular}{|c|c|}
\hline $\begin{array}{l}\text { Zusammenleben im Haushalt, } \mathrm{n} \\
\text { allein } \\
\text { allein mit Kind/Kindern } \\
\text { mit Partner und Kind/Kindern } \\
\text { mit Partner } \\
\text { sonstige }\end{array}$ & $\begin{array}{l}30(30 \%) \\
16(16 \%) \\
34(34 \%) \\
12(12 \%) \\
8(8 \%)\end{array}$ \\
\hline Anzahl der Kinder, Mittelwert (SD), Range & $2,09(1,36), 1-11$ \\
\hline $\begin{array}{l}\text { Alter der Kinder, Mittelwert (SD), Range } \\
\text { Kind } 1(n=100) \\
\text { Kind } 2(n=65) \\
\text { Kind } 3(n=25) \\
\text { Kind } 4(n=11) \\
\text { Kind } 5(n=4)\end{array}$ & $\begin{array}{r}15(9,73), 0,5-48 \\
14(9,66), 0,5-46 \\
12(9,22), 0,5-47 \\
10(4,17), 0,5-17 \\
9,5(0,58), 9-10\end{array}$ \\
\hline $\begin{array}{l}\text { Versorgung Kind unter } \mathbf{1 8 , n} \\
\text { alleine } \\
\text { durch Partner } \\
\text { durch andere } \\
\text { durch mehrere }\end{array}$ & $\begin{array}{c}3(3 \%) \\
44(44 \%) \\
9(9 \%) \\
26(26 \%)\end{array}$ \\
\hline $\begin{array}{l}\text { Versorgung Kind über 18, n } \\
\text { alleine } \\
\text { durch Partner } \\
\text { durch andere } \\
\text { durch mehrere }\end{array}$ & $\begin{array}{c}14(14 \%) \\
14(14 \%) \\
6(6 \%) \\
4(4 \%)\end{array}$ \\
\hline
\end{tabular}

\section{Familiäre Situation und Interventionsbedarf}

Bei 82 \% der Patienten lebte mehr als ein minderjähriges versorgungsbedürftiges Kind im Haushalt, lediglich bei 38 Patienten waren die Kinder, wie aus $>$ Tab. 2 zu entnehmen ist, älter als 18 Jahre. Die Versorgung der Kinder erfolgte in der Mehrheit der Fälle durch den Partner, beteiligt waren hierbei aber auch Großeltern und andere Familienangehörige. Immerhin nahmen 35 Patienten Versorgungsangebote der Jugendhilfe an. Nur 15 der 100 befragten Patienten gaben an, keinerlei Sorgen um die Versorgung der Familienangehörigen zu haben, allerdings gaben lediglich 10 der Patienten klar keinen Interventionsbedarf an.

Bei 71 Patienten bestand neben der Sorge um die Versorgung auch eine Sorge bezüglich der psychischen Entwicklung der Kinder, wobei ein Drittel der Patienten (29,6\%) die Angst vor einer „Weitervererbung“ der psychischen Erkrankung formulierte. Sowohl hinsichtlich der Versorgungssorgen als auch der Sorgen um die psychische Gesundheit der Kinder formulierten $64 \%$ der befragten Patienten den Wunsch nach Hilfs- und Interventionsmöglichkeiten.

\section{Umgang mit dem familiären Interventionsbedarf}

Trotz der bestehenden Sorgen und dem Wunsch nach Hilfe bezüglich der Versorgung der Familienangehörigen, berichteten 74\% der Patienten davon, diese nicht mit dem Therapeuten im Rahmen des aktuellen stationären Aufenthalts thematisiert zu haben. Wesentliche Gründe hierfür waren nach Aussage der Patienten vor allem das diesbezüglich nicht erfolgte Fragen/ Nachfragen des Therapeuten $(n=50)$ sowie die nicht ausreichende Gesprächszeit $(n=7)$. Dies waren auch die Hauptgründe, warum $82 \%$ der Patienten angaben, über ihre Sorgen be-
- Tab. 3 Familiärer Interventionsbedarf der Untersuchungspopulation $(n=100)$.

keine konkrete Idee für eine Hilfe, n $43(43 \%)$

mehr über Versorgungssituation reden, mehr Informationen erhalten, $\mathrm{n}$ $8(8 \%)$

Hilfen bei rechtlicher Regelung der Versorung, $\mathrm{n}$ $12(12 \%)$

Familie in die Therapie miteinbeziehen (Familiengespräche...), n

Hilfen für den Alltag anleiten, $\mathrm{n}$ $11(11 \%)$ $14(14 \%)$

Sozialarbeiter/Sozialdienst/Seelsorge vermitteln, $n$ $12(12 \%)$

züglich der psychischen Entwicklung ihrer Kinder nicht mit dem Therapeuten gesprochen zu haben. Immerhin gab die Mehrheit der Patienten $(n=79)$ an, dass ihr jeweiliger Therapeut in Kenntnis der familiären Situation sei.

In 19\% der Patienten habe es Maßnahmen zur Unterstützung der Versorgung von Familienangehörigen seitens der Therapeuten gegeben, jedoch haben sich insgesamt nur 16 Patienten in ihren Sorgen um die Familienangehörigen vom Therapeuten ernst genommen gefühlt. Auffällig war auch, dass nahezu $40 \%$ der Patienten zu Fragen zu konkreten Unterstützungs- und Verbesserungsmaßnahmen bezüglich des familienmedizinischen Interventionsbedarfs keine Aussagen trafen (z. B. „weiß nicht“, „keine Idee“).

\section{Patientenwünsche bezüglich des Umgangs}

Der Großteil der hier untersuchten Patienten (78\%) war der Auffassung, dass das Wissen um die gute und geregelte Versorgung der Familienangehörigen zur schnellen Genesung beitrage. Die in diesem Kontext stehenden Wünsche der Patienten sind in $>$ Tab. 3 dargestellt.

\section{Korrelative Beziehungen}

Signifikante Zusammenhänge fanden sich zwischen dem Ausmaß der Sorgen bezüglich der Versorgung der Familienangehörigen bereits seit Beginn der psychischen Erkrankung des Befragten und dem Vorliegen einer positiven Familienanamnese $(r=0,263 ; p=0,008)$. Das Vorhandensein von psychosozialen Belastungen korrelierte ebenfalls mit den Versorgungssorgen $(r=0,268 ; p=0,007)$. Darüber hinaus konnte ein Zusammenhang zwischen den Sorgen lediglich für die PSP-Subdimensionen a) (Arbeit, nützliche Aktivitäten; $r=0,207 ; p=0,039$ ) und b) (Beziehungen; $r=0,213 ; p=0,033$ ) gezeigt werden. Eine $a b$ geschlossene Berufsausbildung korrelierte negativ mit den aktuellen Sorgen der Versorgung $(r=-0,260 ; p=0,009)$. Dauer der Erkrankung und Ausmaß der Sorgen seit Beginn der psychischen Erkrankung korrelierten ebenfalls $(r=0,209 ; \quad p=$ 0,037).

Imponierend waren die Zusammenhänge zwischen der Stärke der Versorgungssorgen und den Sorgen um die psychische Entwicklung der Kinder, die sowohl für die gesamte Zeit seit Beginn der psychischen Erkrankung $(r=0,411 ; p \leq 0,000)$ als auch im Vorfeld des stationären Aufenthalts $(r=0,415 ; p \leq 0,000)$ und aktuell zum Zeitpunkt der Befragung $(r=0,304 ; p=0,002)$ 
bestanden. Der Wunsch nach Hilfsmaßnahmen bestand umso häufiger, je größer die Sorgen um die Versorgung $(r=0,236$; $p=0,018$ ) aber auch um die psychische Gesundheit der Angehörigen $(r=0,301 ; p=0,002)$ waren. Schließlich zeigte sich noch eine negative Korrelation zwischen dem Ausmaß der aktuellen Versorgungssorgen und dem Gespräch mit dem Therapeuten über das Kindeswohl $(r=-0,210 ; p=0,036)$.

\section{Diskussion}

In der vorliegenden Untersuchung wurden stationär behandelte Patienten mit einer psychischen Erkrankung zu ihrer familiären Situation und dem subjektiv empfundenen familienmedizinischen Interventionsbedarf befragt. Da alle Patienten Eltern waren, lag das besondere Augenmerk dabei auf Sorgen bezüglich der Versorgung ihrer Kinder und dem Wunsch nach verbesserter Anleitung hinsichtlich möglicher Hilfsmaßnahmen durch die Therapeuten. Die Ergebnisse bestätigten daher, dass die Patienten einen Interventionsbedarf vor allem bezüglich der Versorgung der Kinder formulieren. Lediglich $10 \%$ der Befragten gab klar keinen Interventionsbedarf an. Auch Östman und Hansson stellten 2002 heraus, dass die Mehrheit der Kinder aus Familien mit einem psychisch erkrankten Elternteil einen Bedarf an Unterstützung hatten und Eltern sich häufig Hilfen wünschten [16].

Unsere Befragung deutet an, dass klinisch arbeitende Therapeuten über das Vorhandensein von Kindern Bescheid wissen, eine detaillierte Nachfrage nach der aktuellen Versorgungssituation jedoch von den Patienten als nicht ausreichend angesehen wird. Dies arbeiteten auch Franz et al. 2012 [9] heraus, die Mehrheit der befragten Psychiater wusste, ob ihre Patienten Kinder haben, konnte differenzierte Fragen zur Lebenssituation der Kinder und deren Unterstützungsmöglichkeiten aber nicht beantworten. In dem Zusammenhang interessant ist die Studie mit einem konträren Ergebnis dazu von Wlodarczyk et al., die 2017 [17] in ihrer bundesweiten Befragung von Therapeuten zu dem Ergebnis kam, dass 81,4\% der Fachkräfte angaben, die Versorgungslage der Kinder während des stationären Aufenthalts der Eltern erhoben zu haben.

Insgesamt erachten 74\% der Patienten, dass ihre Sorgen und die Bedürfnisse nach Hilfsleistungen seitens der Therapeuten in Bezug auf die Familie häufig unzureichend thematisiert werden. Zu dem Ergebnis, dass obwohl in den therapeutischen Sitzungen immer wieder „familienorientierte Angebote“ gefordert werden, eine Familienperspektive noch zu wenig Raum findet, kamen auch Lindhorst et al. 2015 [18]. Dass familienorientierte Programme in Deutschland bestehen, zeigten WiegandGrefe et al. 2011 [19]. Sie entwickelten das CHIMPs (Children of mentale ill parents program), ein mögliches familienorientiertes Programm im deutschsprachigen Raum zur Verbesserung der psychisch assoziierten Lebensqualität, Reduktion der mentalen Störungen bei Kindern und Verbesserung der familiären Unterstützung. Auffällig war in unserer Befragung weiterhin, dass $64 \%$ aller Patienten die Frage nach einem Wunsch bezüglich einer Verbesserung der Hilfsmaßnahmen mit „ja“ beantworteten, während ca. 67\% dieser Gruppe keine konkrete Idee in Bezug auf die Art der Hilfe benennen konnte. Coverdale und Long [20] stellten heraus, dass auch in ihrer Untersuchung zum Thema „Emotionales Wohlbefinden und psychische Gesundheit" Befragte berichteten, nicht zu wissen, wo sie Hilfen suchen sollten. Es lässt sich aufgrund der Schwierigkeit für die Patienten, konkrete Hilfestellungen zu benennen und einzufordern, folgern, dass diese wegen der psychischen Situation nicht in der Lage sind, alleine Lösungen zu finden. Der Therapeut ist daher in der Position, die Sorgen aufzugreifen und zu thematisieren sowie mit dem Patienten Lösungsmöglichkeiten zu erarbeiten.

Studien belegen, dass Kinder von psychisch erkrankten Eltern ein höheres Risiko tragen, selbst an psychischen Störungen zu erkranken [21]. So ist dieses Risiko laut Aichhorn et al. [22] im Vergleich zu Kindern psychisch gesunder Eltern bis zu vierfach erhöht. Im Gegensatz dazu stellte die Studie von Festen et al. [23] fest, dass Eltern oft keinen Zusammenhang zwischen der eigenen Erkrankung und Auffälligkeiten aufseiten der Kinder sahen und somit häufig erst das Erfordernis von Hilfen für ihre Kinder sahen, wenn diese schon eine stark ausgeprägte Symptomatik aufwiesen. Dies steht im Kontrast zu der Tatsache, dass mit $71 \%$ auch ein Großteil der Patienten unserer Studie Sorgen um die psychische Entwicklung der Kinder angab und diese sich zu einem Drittel mit einer „Weitervererbung“ der psychischen Krankheit befassten. Diese Auffälligkeit verdeutlicht, dass eine Psychoedukation helfen könnte, sich mit dieser Problematik zu beschäftigen, diesbezüglich Sorgen zu objektivieren und damit zu relativieren. Die Notwendigkeit einer Psychoedukation gerade bei erstmaligem Kontakt mit der Gesundheitsfürsorge betonten auch Chen et al. [24]. Dass ein Mangel an Psychoedukation im stationären klinischen Alltag besteht, stellten Franz et al. [9] heraus. Sie erarbeiteten, dass Ursachen der Erkrankung und deren Verlauf oft nicht erörtert werden. Wir konnten herausstellen, dass besonders dann, wenn starke Sorgen um die psychische Entwicklung der Kinder bestanden, auch die Sorgen um die Versorgung der Kinder besonders hoch waren.

Auffällig war weiterhin, dass eine positive Familienanamnese und das Vorhandensein von psychosozialen Belastungen mit mehr Sorgen um die Versorgung korrelierte. Ebenso empfanden Patienten mit einer langen Krankheitsdauer mehr Sorgen als neu Erkrankte. Es stellt sich die Frage, warum Patienten mit mehr Erfahrung und Aufenthalten in Psychiatrien eine gesteigerte Sorge bezüglich der Familienversorgung empfinden. Dies könnte darauf hinweisen, dass Patienten mit psychiatrischer Anamnese die Erfahrung gemacht haben, dass sich die Versorgung zu Hause während ihres stationären Aufenthalts problematisch gestaltet hat. Diese Hypothese wird gestützt durch die Ergebnisse von Schmid et al. [25], in deren Untersuchung $55 \%$ der Befragten bereits eine stationäre Behandlung abgebrochen oder nicht angetreten hatten, da Sorgen um die Versorgung ihrer Kinder bestanden. Es lässt sich diskutieren, ob die Problematik dadurch zustande kommt, dass es sich bei Patienten mit psychiatrischer Anamnese um ein schwerer erkranktes Patientenkollektiv handelt oder ob die Hilfsangebote tatsächlich mangelhaft sind. 


\section{Fazit und Ausblick}

Zusammenfassend lässt sich sagen, dass sich die Ergebnisse unserer Studie mit denen vieler anderer Studien decken. Es besteht ein familienmedizinischer Interventionsbedarf, stationäre Patienten empfinden die Nachfrage der Therapeuten nach der Versorgung der Kinder als unzureichend und sie wünschen sich eine Verbesserung in den Hilfsmaßnahmen. Der Großteil der Patienten empfindet Sorgen um die psychische Entwicklung der Kinder, besonders eine „Vererbung“ der psychischen Erkrankung wird befürchtet. Die Ergebnisse weisen somit auf eine unzureichende Arzt-Patienten-Interaktion und -Kommunikation sowie Psychoedukation in diesem Bereich hin. Laut der Ottawa-Charta (WHO,1986 [26]) besteht seitens der Hilfssysteme die Pflicht, Missstände in der Behandlung der Kinder psychisch kranker Eltern zu beheben und Versorgungsungleichheiten zu bekämpfen. Somit sollte es für die kommenden Jahre weiterhin das Ziel sein, die Hilfsbedarfe zu erkennen und dadurch möglichst vielen Kindern und Familien Zugang zu Hilfssystemen zu gewähren.

\section{Limitationen der Studie}

Einen wesentlichen limitierenden Faktor im Hinblick auf allgemeingültige Aussagen stellt die Fallzahl von 100 Befragten und die resultierenden kleinen Fallzahlen der Subgruppen dar.

Ebenso muss berücksichtigt werden, dass die Daten stationär behandelter Patienten eher auf ein schwer krankes Patientenklientel verweisen. Zudem liegt eine große Spannweite der Altersklassen der Kinder vor.

Es existieren weder fremdanamnestische Angaben noch wurden die Angaben der Patienten überprüft.

Dennoch deuten die Ergebnisse darauf hin, dass ein familienmedizinischer Interventionsbedarf vorliegt und von der Mehrheit der Patienten ein Wunsch nach Verbesserung der Hilfsmaßnahmen geäußert wird. Es besteht eine Notwendigkeit der Optimierung der Arzt-Patienten-Kommunikation für den Bereich der familiären Versorgung.

\section{KONSEQUENZEN FÜR KLINIK UND PRAXIS}

- Bei stationär behandelten Patienten sollte die Versorgungssituation der Kinder detaillierter erfragt werden.

- Der Therapeut sollte mit dem Patienten konkrete Hilfen für die Versorgung individuell erarbeiten und anleiten.

- Sorgen um die Familie sollten gezielter erfasst und minimiert werden.

\section{Anmerkungen}

Teile der Studienergebnisse entstammen der medizinischen Dissertation von Katharina Zogas.
Interessenkonflikt

Die Autorinnen/Autoren geben an, dass kein Interessenkonflikt besteht.

Literatur

[1] Jacobi F, Höfler M, Siegert J et al. Twelve-month prevalence, comorbidity and correlates of mental disorders in Germany: the Mental Health Module of the German Health Interview and Examination Survey for Adults (DEGS1-MH). Int J Methods Psychiatr Res 2014; 23: 304-319

[2] Gühne U, Becker T, Salitze H] et al. How many people in Germany are seriously mentally ill? Psychiat Prax 2015; 42: 415-423

[3] Griepenstroh J, Schmuhl M. Zur Lebenssituation von Kindern psychisch erkrankter Eltern. Psych Pflege 2010; 16: 123-128

[4] von Kardorff E, Soltaninejad A, Kamali M et al. Family caregiver burden in mental illnesses: The case of affective disorders and schizophrenia - a qualitative exploratory study. Nord J Psychiatry 2016; 70 : 248-254

[5] Monteiro AMF, Santos RL, Kimura N et al. Coping strategies among caregivers of people with Alzheimer disease: a systematic review. Trends Psychiatry Psychother 2018; 40: 258-268

[6] Östman M, Wallsten T, Kjellin L. Family burden and relatives' participation in psychiatric care: are the patient's diagnosis and the relation to the patient of importance? Int J Soc Psychiatry 2005; 51: 291-301

[7] Stålberg G, Ekerwald H, Hultman CM. At issue: siblings of patients with schizophrenia: sibling bond, coping patterns, and fear of possible schizophrenia heredity. Schizophr Bull 2004; 30: 445-458

[8] Howard L, Underdown $\mathrm{H}$. The needs of mentally ill parents - a review of the literature. Psychiat Prax 2011; 38: 8-15

[9] Franz M, Kettemann B, Jäger K et al. Was wissen Psychiater über die Kinder ihrer Patienten? Psychiat Prax 2012; 39: 211-216

[10] Solantaus T, Paavonen EJ, Toikka $S$ et al. Preventive interventions in families with parental depression: children's psychosocial symptoms and prosocial behaviour. Eur Child Adolesc Psychiatry 2010; 19: 883892

[11] Dincin J, Selleck V, Streicker S. Restructuring parental attitudes working with parents of the adult mentally ill. Schizophr Bull 1978; 4: $597-608$

[12] Shpigner E, Possick C, Buchbinder E. Parents' experience of their child's first psychiatric breakdown: "welcome to hell". Soc Work Health Care 2013; 52: 538-557

[13] Pilling S, Bebbington P, Kuipers E et al. Psychological treatments in schizophrenia: I. Meta-analysis of family intervention and cognitive behaviour therapy. Psychol Med 2002; 32: 783-791

[14] Guy W, ed. Clinical Global Impression (CGI). ECDEU Assessment Manual for Psychopharmacology. Rockville, MD: U.S. Department of Health, Education, and Welfare; 1976

[15] Juckel G, Schaub D, Fuchs $N$ et al. Validation of the Personal and Social Performance (PSP) Scale in a German sample of acutely ill patients with schizophrenia. Schizophr Res 2008; 104: 287-293

[16] Östman M, Hansson L. Children in families with a severely mentally ill member. Prevalence and needs for support. Soc Psychiatry Psychiatr Epidemiol 2002; 37: 243-248

[17] Wlodarczyk O, Metzner F, Pawils S. Befragung zur Versorgungssituation und -hindernissen von Kindern psychisch kranker Eltern aus Sicht der Erwachsenenpsychiatrie. Psychiat Prax 2017; 44: 393-399

[18] Lindhorst K, Bauer U, Osipov I et al. Kanu - Gemeinsam weiterkommen. Ein Projekt zur primären Prävention psychischer Erkrankungen 
bei Kindern psychisch kranker Eltern. Gesundheitswesen 2015; 77: S133-S134

[19] Wiegand-Grefe S, Halverscheid S, Olass A. Kinder und ihre psychisch kranken Eltern: familienorientierte Prävention - der CHIMPs - Beratungsansatz. Göttingen: Hogrefe; 2011

[20] Coverdale E, Long A. Emotional wellbeing and mental health: an exploration into health promotion in young people and families. Perspect Public Health 2015; 135: 27-36

[21] Mattejat F, Remschmidt H. Kinder psychisch kranker Eltern. Dtsch Ärtzebl 2008; 7: 312-317

[22] Aichhorn W, Stelzing-Schöler R, Hasselbring L et al. Häufigkeit und Risikofaktoren für psychische Auffälligkeiten bei Kindern psychiatrischer Patienten. Neuropsychiatrie 2011; 25: 192-198
[23] Festen H, Schipper K, de Vries SO et al. Parents' perceptions on offspring risk and prevention of anxiety and depression: a qualitative study. BMC Psychol 2014; 2: 17

[24] Chen F, Gearing R, DeVylder J et al. Pathway model of parental help seeking for adolescents experiencing first-episode psychosis. Early Interv Psychiatry 2016; 10: 122-128

[25] Schmid M, Schielke A, Becker T et al. Versorgungssituation von Kindern während einer stationären psychiatrischen Behandlung ihrer Eltern. Nervenheilkunde 2008; 27: 533-539

[26] WHO (World Health Organization). The Ottawa-Charta for Health Promotion. 1986: Zugriff unter: https://www.who.int/healthpromotion/conferences/previous/ottawa/en/ (24.05.2019) 